\title{
Development of Transmitting Boundary for Periodically Supported Rails*
}

\author{
Kazuhisa $\mathrm{ABE}^{* *}$, Shingo FUJISHITA ${ }^{* * *}$ and Kazuhiro KORO** \\ ** Niigata University \\ 8050 Igarashi 2-Nocho, Nishi-ku, Niigata 950-2181, Japan \\ E-mail: abe@eng.niigata-u.ac.jp \\ *** Niigata City Government \\ 602-1 Gakkocho 1-Bancho, Chuo-ku, Niigata 951-8550, Japan
}

\begin{abstract}
This paper presents a numerical method to construct transmitting boundaries for periodically supported railway tracks. This is realized by the derivation of impedance matrix for a half-infinite track. To achieve this, dynamic response of the infinite track corresponding to the half-infinite model is analyzed for a series of harmonic loading modes. The infinite problems are solved by virtue of Floquet transform. The developed method is applied to a periodically supported rail having a finite irregular zone subjected to harmonic loads. It is reduced to a dynamic problem of a finite track with the transmitting boundaries attached on both ends. Through comparison with the present method and a conventional way using a very long but finite railway model, the superiority over the truncated model is demonstrated.
\end{abstract}

Key words : Transmitting Boundary, Periodic Railway Track, Irregular Zone, Floquet Transform

\section{Introduction}

To understand the dynamic behavior of railway tracks is important from the viewpoint of vibration reduction and maintenance of tracks. For this purpose, various dynamic analyses have been attempted ${ }^{(1)}$. The majority of those numerical methods are applicable only for finite rails even if the mathematical model of track is given by an infinitely long structure. While the integral representation method with the Green's function ${ }^{(2),(3)}$ enables to describe the dynamic deflection of an infinite beam, discrete supports are to be allocated within a finite region. Therefore, for that formulation, it is essentially impossible to capture the infinity.

In the case of dynamic analysis for tracks with damping, since waves reflected from the ends may decay and not reach an interesting range, the harm due to the truncation will be negligible. Thus, in practice, numerical methods with finite domain are applicable to quantitative simulation of track vibrations. On the other hand, the dynamic response of undamped system can also be helpful to understand the fundamental features of track vibrations. However, in this case waves reflected from the ends may propagate in the rail without any dissipation and the existence of truncated ends will affect the responses. Consequently, a finite model cannot be an approximation of infinite one.

In general, a railway track can be modeled as an infinite periodic structure in which the periodicity is characterized by sleepers. Dynamic behavior of periodic structures can be analyzed by virtue of the Floquet's theorem ${ }^{(4)}$. For example, wave propagation in an infinite rail supported by discrete sleepers was investigated by the aid of the theorem ${ }^{(5)}$. In that case the problem is reduced to the one in a unit cell representing the periodicity. Owing to this the discretization of only one unit cell is required in the analysis. However, the method is valid only for fully periodicity. If such irregularity as a rail joint exists in the structure, the theorem is no longer applicable. In spite of this, since the irregular zone can be a weak portion, it is

Received 28 0ct., 2009 (No. 09-0632) [DOI: 10.1299/jmtl.3.44]

Copyright (c) 2010 by JSME 


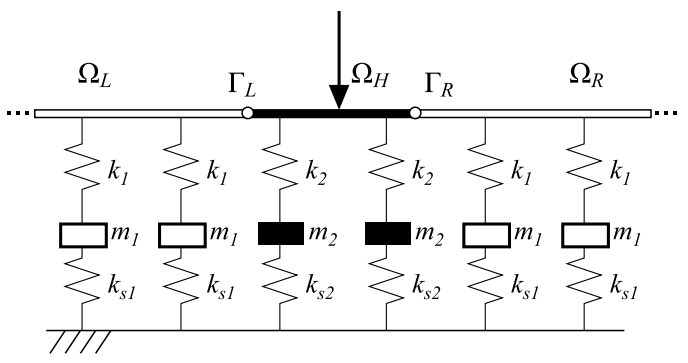

Fig. 1 Infinite track having an irregular zone

important to assess the dynamic behavior precisely.

To cope with this difficulty, in this paper the derivation of transmitting boundaries is attempted for periodic railway structures. The construction of nonreflecting boundary is realized within the framework of the Floquet transform ${ }^{(6)}$. The infinite periodic structure including a hetero-region can then be analyzed by allocating the transmitting boundaries to both ends of the irregular zone. Therefore, the dynamic problem of an infinite railway track is reduced to the vibration analysis of a finite region. Through numerical examples, the efficiency of the method is demonstrated.

Nomenclature

$\omega:$ circular frequency, $\mathrm{rad} / \mathrm{s}$

$\kappa:$ Floquet wavenumber, $\mathrm{rad} / \mathrm{m}$

$L:$ periodic length of railway track, $\mathrm{m}$

$\mathbf{K}$ : stiffness matrix

\author{
$\mathbf{M}$ : mass matrix \\ $\mathbf{U}$ : nodal displacement vector \\ $\mathbf{F}$ : nodal force vector \\ $\check{\mathbf{K}}$ : impedance matrix
}

\section{Outline of the Method}

Let us consider an infinitely long track consisted of a finite irregular zone $\Omega_{H}$, and halfinfinite periodic regions $\Omega_{L}$ and $\Omega_{R}$ lying on the left- and right-hand sides (Fig.1). The interfaces of $\Omega_{L}-\Omega_{H}$ and $\Omega_{R}-\Omega_{H}$ are denoted by $\Gamma_{L}$ and $\Gamma_{R}$, respectively. We assume that the system is given by an undamped structure and harmonic loads are acting only on $\Omega_{H}$.

The equation of motion of the substructure in $\Omega_{H}$ is given by

$$
\left[\begin{array}{ccc}
\hat{\mathbf{K}}_{L L} & \hat{\mathbf{K}}_{L H} & \hat{\mathbf{K}}_{L R} \\
\hat{\mathbf{K}}_{H L} & \hat{\mathbf{K}}_{H H} & \hat{\mathbf{K}}_{H R} \\
\hat{\mathbf{K}}_{R L} & \hat{\mathbf{K}}_{R H} & \hat{\mathbf{K}}_{R R}
\end{array}\right]\left\{\begin{array}{c}
\mathbf{U}_{L} \\
\mathbf{U}_{H} \\
\mathbf{U}_{R}
\end{array}\right\}=\left\{\begin{array}{c}
\mathbf{F}_{L} \\
\overline{\mathbf{F}}_{H} \\
\mathbf{F}_{R}
\end{array}\right\},
$$

where $\hat{\mathbf{K}}_{i j}=\mathbf{K}_{i j}-\omega^{2} \mathbf{M}_{i j}, \mathbf{K}_{i j}$ and $\mathbf{M}_{i j}$ are sub-matrices of the stiffness and mass matrices, and $\omega$ is the circular frequency. $\{\mathbf{U}\}$ and $\{\mathbf{F}\}$ are nodal displacement and force vectors. ()$_{L}$ and ()$_{R}$ stand for sub-vectors associating to nodes on $\Gamma_{L}$ and $\Gamma_{R}$. ( ) $)_{H}$ is a sub-vector corresponding to nodes in $\Omega_{H} \backslash\left(\Gamma_{L} \cup \Gamma_{R}\right) .\left\{\mathbf{F}_{L}\right\}$ and $\left\{\mathbf{F}_{R}\right\}$ are defined by internal forces and thus unknowns. $\left\{\overline{\mathbf{F}}_{H}\right\}$ is given by prescribed external loads.

Suppose that, for both half-infinite tracks, we have relations between displacement $\{\mathbf{U}\}$ and force $\{\mathbf{F}\}$ on the boundaries of $\Gamma_{L}$ and $\Gamma_{R}$ as

$$
\begin{aligned}
& {\left[\check{\mathbf{K}}_{L}\right]\left\{\mathbf{U}_{L}\right\}=\left\{\mathbf{F}_{L L}\right\} \quad\left(\text { on } \Gamma_{L}\right),} \\
& {\left[\check{\mathbf{K}}_{R}\right]\left\{\mathbf{U}_{R}\right\}=\left\{\mathbf{F}_{R R}\right\} \quad\left(\text { on } \Gamma_{R}\right) \text {, }}
\end{aligned}
$$

where $\left\{\mathbf{F}_{L L}\right\}$ and $\left\{\mathbf{F}_{R R}\right\}$ are nodal forces on $\Gamma_{L}$ of $\Omega_{L}$ and $\Gamma_{R}$ of $\Omega_{R}$, [ $\left.\check{\mathbf{K}}_{L}\right]$ and $\left[\check{\mathbf{K}}_{R}\right]$ are impedance matrices on $\Gamma_{L}$ and $\Gamma_{R}$, respectively. The equilibrium conditions on $\Gamma_{L}$ and $\Gamma_{R}$ are given by

$$
\begin{array}{ll}
\left\{\mathbf{F}_{L}\right\}+\left\{\mathbf{F}_{L L}\right\}=0 & \left(\text { on } \Gamma_{L}\right), \\
\left\{\mathbf{F}_{R}\right\}+\left\{\mathbf{F}_{R R}\right\}=0 & \left(\text { on } \Gamma_{R}\right) .
\end{array}
$$




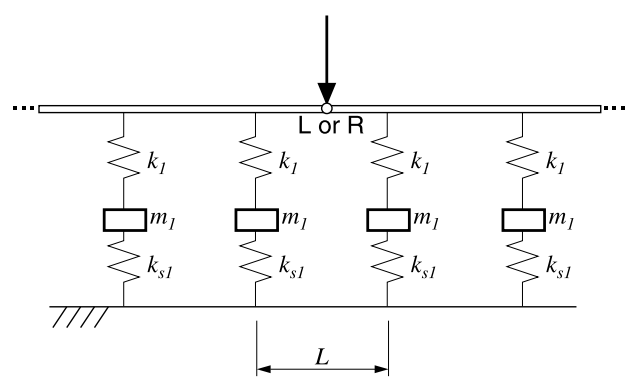

Fig. 2 Periodic track subjected to a harmonic load

Imposing the equilibrium conditions on Eqs.(1) and (2), the equation of motion of the infinite structure can then be obtained by

$$
\left[\begin{array}{ccc}
\hat{\mathbf{K}}_{L L}+\check{\mathbf{K}}_{L} & \hat{\mathbf{K}}_{L H} & \hat{\mathbf{K}}_{L R} \\
\hat{\mathbf{K}}_{H L} & \hat{\mathbf{K}}_{H H} & \hat{\mathbf{K}}_{H R} \\
\hat{\mathbf{K}}_{R L} & \hat{\mathbf{K}}_{R H} & \hat{\mathbf{K}}_{R R}+\check{\mathbf{K}}_{R}
\end{array}\right]\left\{\begin{array}{c}
\mathbf{U}_{L} \\
\mathbf{U}_{H} \\
\mathbf{U}_{R}
\end{array}\right\}=\left\{\begin{array}{c}
\mathbf{0} \\
\overline{\mathbf{F}}_{H} \\
\mathbf{0}
\end{array}\right\} .
$$

Notice that we can attain the dynamic response of the infinite system by solving the above matrix equation with finite DOF.

\section{Derivation of Transmitting Boundary}

\subsection{Harmonic Loading of Periodic Structure}

The dynamic analysis described in the preceding section is reduced to the development of the impedance matrices $\left[\check{\mathbf{K}}_{L}\right]$ and $\left[\check{\mathbf{K}}_{R}\right]$ by which the transmitting boundaries are realized. Transmitting boundaries for waves propagating parallel to layered media have been developed by Lysmer and Waas ${ }^{(7)}$, and are now widely utilized in various problems. A similar problem has first been discussed theoretically for a periodic structure by Brillouin ${ }^{(8)}$, and a numerical method has been proposed by Lie and Tanabe ${ }^{(9)}$. However, application of the latter method is restricted to a very simple structure with small DOF. In this study a new transmitting boundary which is applicable to any periodic structures is proposed within the framework of finite element analysis.

In order to derive $\left[\check{\mathbf{K}}_{L}\right]$ and $\left[\check{\mathbf{K}}_{R}\right]$, we consider an infinite structure having the same periodicity as $\Omega_{L}$ or $\Omega_{R}$. The structure is subjected to a harmonic load acting on the node corresponding to $\Gamma_{L}$ or $\Gamma_{R}$ as shown in Fig.2. The nodal displacement and stress (internal forces) vectors at the loading point are designated by $\left\{\mathbf{U}_{i}\right\}$ and $\left\{\mathbf{F}_{i}\right\}$, where the subscript ' $i$ ' stands for the $i$ th loading mode. The stresses are to be evaluated in the left side element of the loading node for derivation of $\left[\check{\mathbf{K}}_{L}\right]$, while the stresses in the right side element are to be considered for $\left[\check{\mathbf{K}}_{R}\right]$. Assembling vectors $\left\{\mathbf{U}_{i}\right\}$ and $\left\{\mathbf{F}_{i}\right\}$ for all modes, we can obtain square matrices [ $\left.\check{\mathbf{U}}\right]$ and $[\check{\mathbf{F}}]$ defined as

$$
\begin{aligned}
& {[\check{\mathbf{U}}]=\left[\mathbf{U}_{1} \mathbf{U}_{2} \cdots \mathbf{U}_{N}\right],} \\
& {[\check{\mathbf{F}}]=\left[\mathbf{F}_{1} \mathbf{F}_{2} \cdots \mathbf{F}_{N}\right] \text {, }}
\end{aligned}
$$

where $N$ is the number of loading modes, and these modes are independent of each other. For Bernoulli-Euler beam model two independent modes $(N=2)$ can be given by a vertical force and a moment.

From Eq.(5) the impedance matrices are implicitly defined by

$$
\begin{aligned}
{\left[\check{\mathbf{K}}_{L}\right]\left[\check{\mathbf{U}}_{L}\right] } & =\left[\check{\mathbf{F}}_{L}\right], \\
{\left[\check{\mathbf{K}}_{R}\right]\left[\check{\mathbf{U}}_{R}\right] } & =\left[\check{\mathbf{F}}_{R}\right] .
\end{aligned}
$$

Therefore $\left[\check{\mathbf{K}}_{L}\right]$ and [ $\left.\check{\mathbf{K}}_{R}\right]$ can be obtained by solving Eq.(6), i.e.,

$$
\begin{aligned}
{\left[\check{\mathbf{K}}_{L}\right] } & =\left[\check{\mathbf{F}}_{L}\right]\left[\check{\mathbf{U}}_{L}\right]^{-1}, \\
{\left[\check{\mathbf{K}}_{R}\right] } & =\left[\check{\mathbf{F}}_{R}\right]\left[\check{\mathbf{U}}_{R}\right]^{-1} .
\end{aligned}
$$




\subsection{Floquet Transform}

From Eqs.(5) and (7), the construction of the impedance matrices $\left[\check{\mathbf{K}}_{L}\right]$ and $\left[\check{\mathbf{K}}_{R}\right]$ is reduced to the calculation of $\left\{\mathbf{U}_{i}\right\}$ and $\left\{\mathbf{F}_{i}\right\}$ for the periodic structures. To achieve this, the dynamic response for harmonic loading is analyzed by means of the Floquet analysis. In the following, the outline of the Floquet transform ${ }^{(6)}$ is described.

Let us consider a function $f(x)$ of $x \in \mathbb{R}$. The Floquet transform $\tilde{f}$ with a size $L$ is given by

$$
\tilde{f}(\tilde{x}, \kappa)=\sum_{n=-\infty}^{\infty} f(\tilde{x}+n L) e^{i n \kappa L},
$$

where $i=\sqrt{-1},-L / 2 \leq \tilde{x} \leq L / 2,0 \leq \kappa \leq 2 \pi / L$, and $\kappa$ is called Floquet wavenumber. From the above equation we can readily understand that $\tilde{f}$ has the following periodicity,

$$
\begin{aligned}
& \tilde{f}(\tilde{x}+L, \kappa)=e^{-i \kappa L} \tilde{f}(\tilde{x}, \kappa), \\
& \tilde{f}\left(\tilde{x}, \kappa+\frac{2 \pi}{L}\right)=\tilde{f}(\tilde{x}, \kappa) .
\end{aligned}
$$

Consider a dynamic problem given by the following equation of motion

$$
\mathcal{L}(x) u(x)=f(x)
$$

where $\mathcal{L}$ is an operator associated with the present dynamic problem, $u(x)$ is the displacement and $f(x)$ is the external force. Suppose that the structure has the periodicity of $L$, while $u(x)$ and $f(x)$ are nonperiodic functions.

From Eq.(8) the floquet transform of Eq.(11) is described by

$$
\sum_{n=-\infty}^{\infty} \mathcal{L}(\tilde{x}+n L) u(\tilde{x}+n L) e^{i n \kappa L}=\sum_{n=-\infty}^{\infty} f(\tilde{x}+n L) e^{i n \kappa L} .
$$

Due to the periodicity of the structure, $\mathcal{L}$ satisfies the relation

$$
\mathcal{L}(\tilde{x}+n L)=\mathcal{L}(\tilde{x}) .
$$

Therefore Eq.(12) can be rewritten as

$$
\mathcal{L}(\tilde{x}) \tilde{u}(\tilde{x}, \kappa)=\tilde{f}(\tilde{x}, \kappa) .
$$

The Floquet transform $\tilde{u}$ can be obtained by solving the dynamic problem of Eq.(14) for a unit cell characterized by $L$ under the periodicity of Eq.(9). The displacement $u(x)$ in the infinite region is reproduced by the inverse Floquet transform :

$$
u(x)=\frac{L}{2 \pi} \int_{0}^{2 \pi / L} \tilde{u}(\tilde{x}, \kappa) e^{-i n \kappa L} d \kappa,
$$

where $x=\tilde{x}+n L$.

The analysis procedure mentioned above enables us to obtain the response of the periodic structure shown in Fig. 2 by solving dynamic problems of the unit cell for each Floquet wavenumber $\kappa$ and performing the inverse Floquet transform. We can then extract $\left\{\mathbf{U}_{i}\right\}$ and $\left\{\mathbf{F}_{i}\right\}$ from the solution.

\subsection{Floquet Analysis of Track Model}

The unit cell of the periodic track is given by a substructure as shown in Fig.3. For the Floquet transform the equation of motion of the unit cell is given by

$$
\left[\overline{\tilde{\mathbf{V}}}_{l} \overline{\mathbf{\mathbf { V }}}_{m} \overline{\mathbf{\mathbf { V }}}_{r}\right]^{T}\left[\begin{array}{ccc}
\hat{\mathbf{K}}_{l l} & \hat{\mathbf{K}}_{l m} & \hat{\mathbf{K}}_{l r} \\
\hat{\mathbf{K}}_{m l} & \hat{\mathbf{K}}_{m m} & \hat{\mathbf{K}}_{m r} \\
\hat{\mathbf{K}}_{r l} & \hat{\mathbf{K}}_{r m} & \hat{\mathbf{K}}_{r r}
\end{array}\right]\left\{\begin{array}{c}
\tilde{\mathbf{U}}_{l} \\
\tilde{\mathbf{U}}_{m} \\
\tilde{\mathbf{U}}_{r}
\end{array}\right\}=\left[\overline{\tilde{\mathbf{V}}}_{l} \overline{\tilde{\mathbf{V}}}_{m} \overline{\mathbf{V}}_{r}\right]^{T}\left\{\begin{array}{c}
\tilde{\mathbf{F}}_{l} \\
\tilde{\mathbf{F}}_{m} \\
\tilde{\mathbf{F}}_{r}
\end{array}\right\},
$$

where ()$_{l}$ and ()$_{r}$ are sub-vectors corresponding to nodes on the left and right ends, respectively. ( $)_{m}$ stands for a sub-vector associating to nodes of the rest. $\{\tilde{\mathbf{V}}\}$ is the variation of 


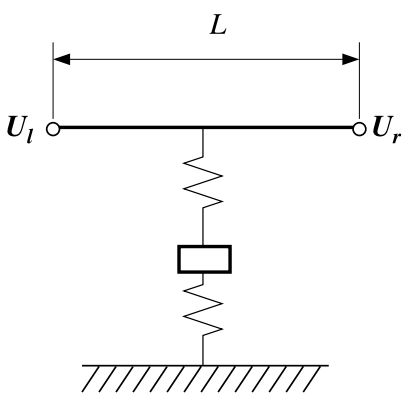

Fig. 3 Unit cell of periodic track

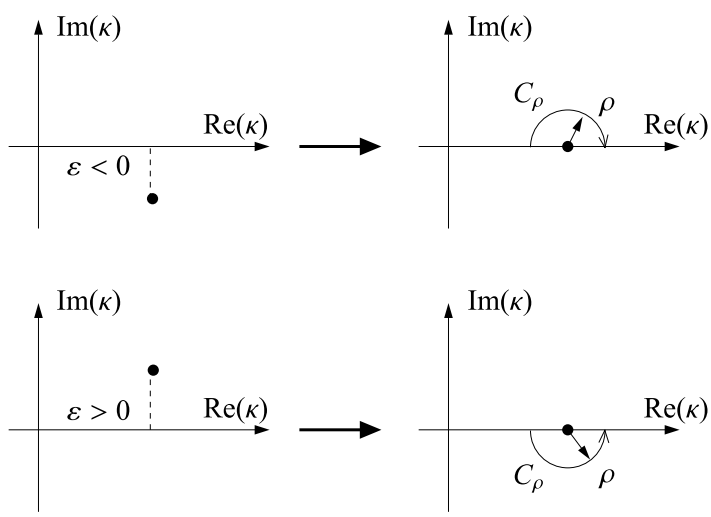

Fig. 4 Path of integration

nodal displacement. ()$^{T}$ denotes the transpose. $\left(^{-}\right)$is the conjugate. Due to the periodicity of Eq.(9), for the Floquet transform with respect to $\kappa$, the following relations are satisfied

$$
\left\{\tilde{\mathbf{U}}_{r}\right\}=e^{-i \kappa L}\left\{\tilde{\mathbf{U}}_{l}\right\}, \quad\left\{\overline{\tilde{\mathbf{V}}}_{r}\right\}=e^{i \kappa L}\left\{\overline{\tilde{\mathbf{V}}}_{l}\right\}, \quad\left\{\tilde{\mathbf{F}}_{r}\right\}=-e^{-i \kappa L}\left\{\tilde{\mathbf{F}}_{l}\right\} .
$$

Substituting Eq.(17) into Eq.(16), we can obtain the equation of motion as

$$
\left[\begin{array}{cc}
\hat{\mathbf{K}}_{l l}+\hat{\mathbf{K}}_{r r}+\hat{\mathbf{K}}_{l r} \cos \kappa L & \hat{\mathbf{K}}_{l m}+\hat{\mathbf{K}}_{r m} e^{i \kappa L} \\
\hat{\mathbf{K}}_{m l}+\hat{\mathbf{K}}_{m r} e^{-i k L} & \hat{\mathbf{K}}_{m m}
\end{array}\right]\left\{\begin{array}{c}
\tilde{\mathbf{U}}_{l} \\
\tilde{\mathbf{U}}_{m}
\end{array}\right\}=\left\{\begin{array}{c}
\mathbf{0} \\
\tilde{\mathbf{F}}_{m}
\end{array}\right\} .
$$

The loading mode stated in $\mathbf{3 . 1}$ is given by a delta function. Since the Floquet transform of delta function is given by the delta function, the force vector $\left\{\tilde{\mathbf{F}}_{m}\right\}$ in Eq.(18) is identical with the original loading mode. Solution of Eq.(18) gives the Floquet transform $\left\{\tilde{\mathbf{U}}_{i}\right\}$ for the $i$ th loading mode.

\subsection{Implementation of Inverse Floquet Transform}

Once the Floquet transform is obtained, we can reproduce the response of the infinite structure by way of the inverse Floquet transform. That given by Eq.(15) is achieved through an integration with respect to the Floquet wavenumber $\kappa$. Since the integrand $\tilde{u}$ is to be obtained by solving the finite element equation corresponding to Eq.(18), the integration in Eq.(15) should be calculated numerically. When the structure possesses some damping, the finite element equation has no singularity. Therefore the numerical integration can be achieved without any difficulty. In contrast to this, the matrix equation may have singularities for no damping systems. If the frequency lies in a band in which propagating wave modes are existing, the integration must encounter the singularity. Moreover, its singularity is strong, i.e., the integration should be interpreted in the context of Cauchy's integral theorem.

To achieve this, the integral domain of $\kappa$ is extended to the complex plane. Then the dissipation is introduced in the structure by replacing the stiffness $E$ to a complex one $(1+i \alpha) E$. 


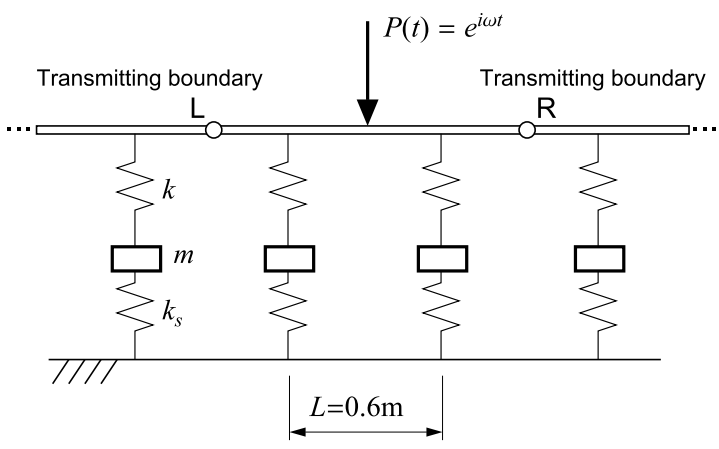

Fig. 5 Analytical conditions of periodic track subjected to a harmonic load

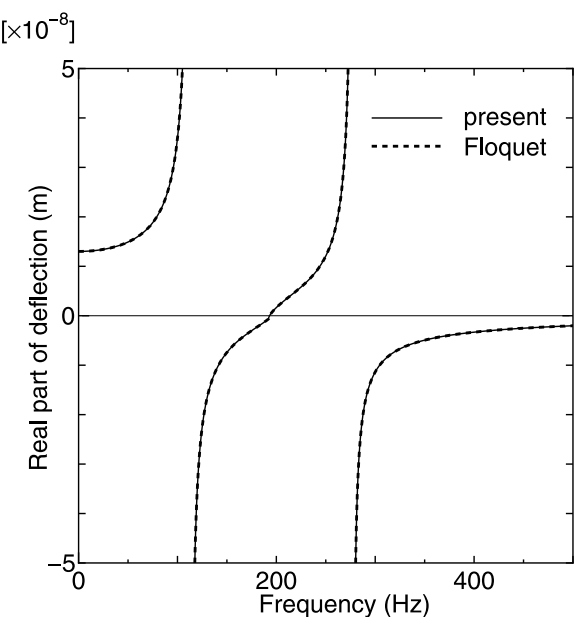

Fig. 6 Real part of deflection at the loading point

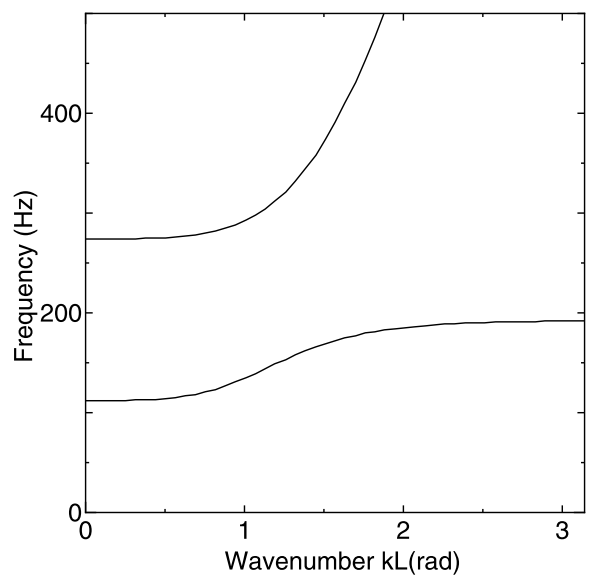

Fig. 7 Dispersion curves of wave propagation modes

Here $\alpha$ is a positive real number. The inverse Floquet transform is re-defined as

$$
u(x)=\lim _{\alpha \rightarrow 0} \frac{L}{2 \pi} \int_{0}^{2 \pi / L} \tilde{u}(\tilde{x}, \kappa ; \alpha) e^{-i n \kappa L} d \kappa,
$$

where $\tilde{u}(\tilde{x}, \kappa ; \alpha)$ is displacement with a complex stiffness. For $\alpha>0$, the singular point is offset from the real axis. In order to attain the same result as Eq.(19), the path of integration for $\alpha=0$ is chosen as shown in Fig.4 based on the location of the singular point for $\alpha>0$.

When the singular point is shifted to the lower half-plane $(\varepsilon<0)$ due to the introduction of positive damping, a semicircular path $C_{\rho}$ of radius $\rho$ is inserted in the upper half-plane as illustrated in Fig.4. The sign of $\varepsilon$ at each singular point is decided based on the slope of dispersion curves in $\kappa-\omega$ space as deduced in Ref. (10). Namely, $\varepsilon<0$ for ascending slope, while $\varepsilon>0$ for descending slope. The numerical integration by Gaussian quadrature is performed on each interval divided by $C_{\rho}$. The larger the radius $\rho$, the better the accuracy of integration, because of the reduction of near singularity. On the other hand, in order to exclude poles from the semicircular domain bounded by $C_{\rho}, \rho$ should be small enough. In the following analyses $\rho$ is set to $\pi / 20 L$.

\section{Numerical Examples}

\subsection{Harmonic Loading of Periodic Railway Track}

To verify the feasibility of the developed method, a periodic railway track subjected to a harmonic load is analyzed. The analytical conditions are shown in Fig.5. Since the structure has fully periodicity of $L=0.6 \mathrm{~m}$, the dynamic response can be obtained directly through the Floquet analysis without the transmitting boundaries. The track is consisted of an infinite rail of JIS $50 \mathrm{kgN}$ and sleepers of $80 \mathrm{~kg}$. Stiffness of the pads is $k=k_{s}=60 \mathrm{MN} / \mathrm{m}$. In the 


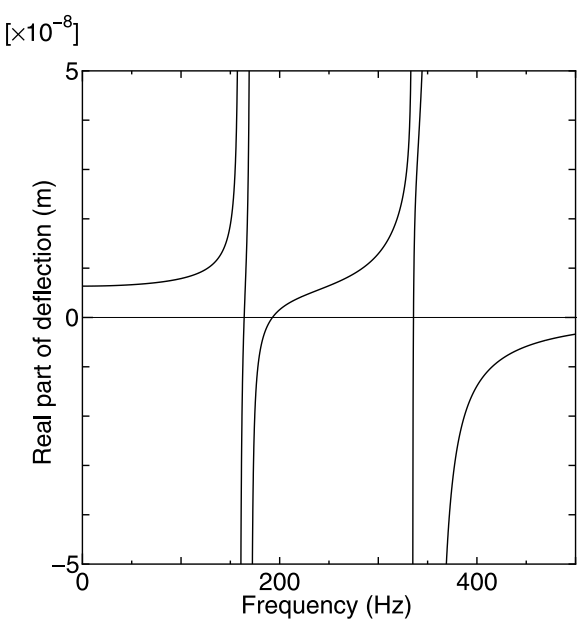

Fig. 8 Real part of deflection at the loading point (Finite model with 4 spans)

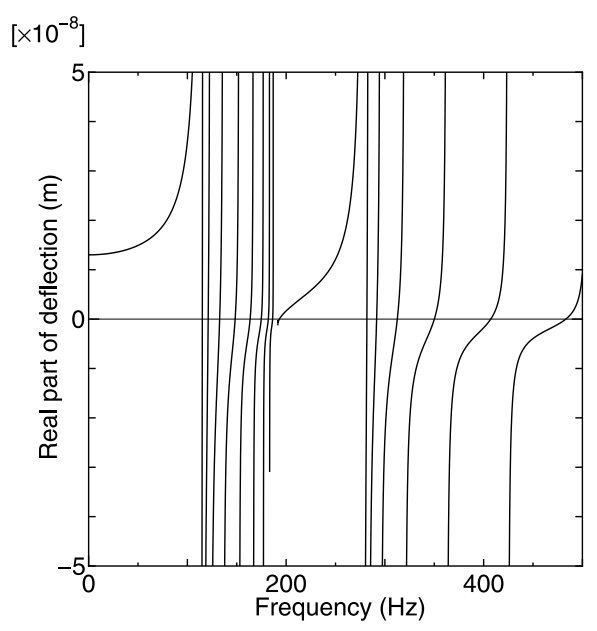

Fig. 9 Real part of deflection at the loading point (Finite model with 30 spans)

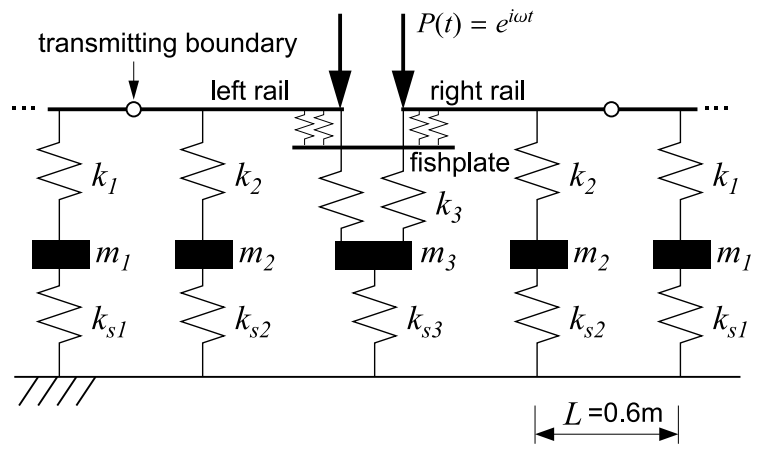

Fig. 10 Infinite rail with a rail joint

analysis the rail is modeled by a Bernoulli-Euler beam and the sleeper is represented by mass. Since the periodicity is characterized by the sleeper space, a unit cell is set as shown in Fig.3. The rail in the unit cell is divided into 16 beam elements, and then the Floquet analysis is performed. Once the transmitting boundaries are constructed, those are attached on both ends of a truncated model. In this analysis the finite track of $2 L$ is employed (Fig.5). Under this condition, a unit harmonic load acting on the mid-span is considered.

Real part of deflection at the loading point is shown in Fig.6. In the figure the response given by the direct analysis with the Floquet transform is also shown. Validity of the proposed method can be evidenced by good agreement between the present method and the direct analysis.

The dispersion curves of wave propagation modes ${ }^{(5)}$ in the present track model are given in Fig.7. A frequency range in which a dispersion curve is lying is called pass band. From the figure, it can be understood that the resonance in Fig.6 is corresponding to the standing wave modes at the lower ends of pass bands. The standing wave at frequency of about $200 \mathrm{~Hz}$ has a node at the loading point. Therefore, the response for this mode is vanished as in Fig.6.

Responses given by a conventional finite element analysis with finite track model of 4 and 30 spans are shown in Figs.8 and 9. In contrast with the response in Fig.6, many resonance frequencies are observed. This anomaly claims that finite track models cannot capture the dynamic modes of infinite rail. From these figures it can also be found that the longer the finite rail, the more complexity the response has.

\subsection{Infinite Rail with a Rail Joint}

A jointed infinite rail is considered as shown in Fig.10. A couple of harmonic forces 
Table 1 Material properties in the jointed rail model

\begin{tabular}{lr|r}
\hline$k_{1}$ & $\mathrm{MN} / \mathrm{m}$ & 110 \\
$k_{2}$ & $\mathrm{MN} / \mathrm{m}$ & 47.3 \\
$k_{3}$ & $\mathrm{MN} / \mathrm{m}$ & 35.5 \\
$k_{s 1}$ & $\mathrm{GN} / \mathrm{m}$ & 10 \\
$k_{s 2}$ & $\mathrm{MN} / \mathrm{m}$ & 133.3 \\
$k_{s 3}$ & $\mathrm{MN} / \mathrm{m}$ & 200 \\
\hline$m_{1}$ & $\mathrm{~kg}$ & 80 \\
$m_{2}$ & $\mathrm{~kg}$ & 43.75 \\
$m_{3}$ & $\mathrm{~kg}$ & 117.58 \\
\hline
\end{tabular}

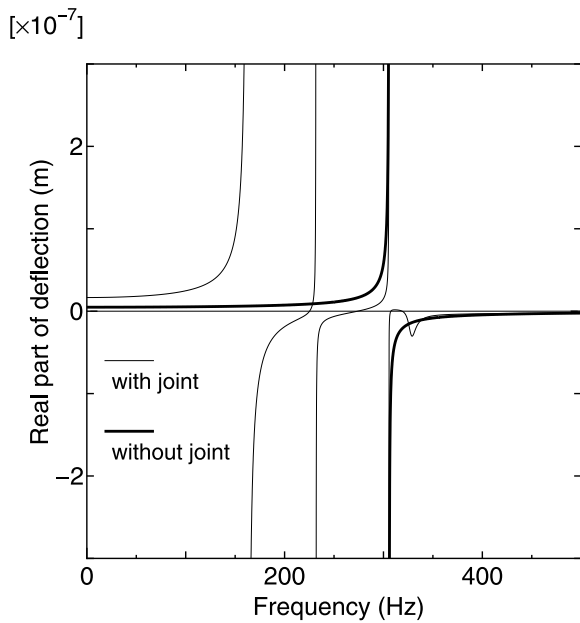

Fig. 11 Real part of deflection at the rail joint

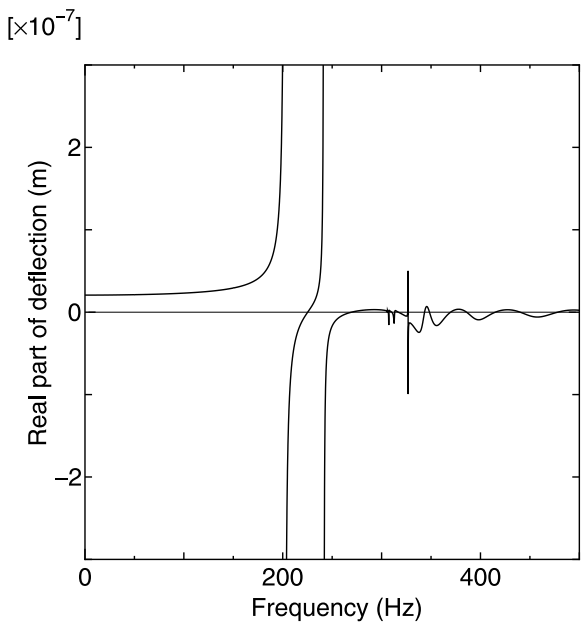

Fig. 12 Real part of deflection at the rail joint (Finite model with 30 spans)

given by $P(t)=e^{i \omega t}$ are acting on the rail ends at the rail joint. Material properties in the model are listed in Table 1. The finite region of the track including the fishplate and three sleepers is discretized. The transmitting boundaries are attached on the both of truncated ends.

The real part of deflection at the rail joint is shown in Fig.11 at frequencies lower than $500 \mathrm{~Hz}$. In the figure the response of an infinite track having identical periodicity with the half-infinite track on both sides is also shown. It is found that two resonance frequencies take place at $160 \mathrm{~Hz}$ and $230 \mathrm{~Hz}$ due to the introduction of the rail joint.

The same problem is analyzed by a finite model truncated with 30 spans. The result is shown in Fig.12. The values and the number of resonance frequencies are completely different from those in Fig.11. From the figure, we can see that the increase of the number of spans cannot improve the approximation, and the consideration of infinity is essential to understand fundamental properties.

\section{Conclusion}

In this paper a transmitting boundary was developed for periodic railway tracks. The derivation of that impedance matrix was accomplished by the aid of the Floquet transform. The method makes it possible to analyze an infinite track having an irregular zone by attaching the transmitting boundaries on both ends of the finite region. The proposed approach was applied to dynamic response analyses of railway tracks. It is found that the present method can precisely represent the infinity in periodic tracks. On the other hand, the conventional model with finite region cannot capture the dynamic characteristics of undamped infinite system. From these results it is revealed that the consideration of the periodical infinity is necessary to extract fundamental properties in track vibrations, and the proposed method can be a useful tool for this purpose. Although, in this study, the loading portion is restricted to the inside of the irregular zone, this condition can be eased within the framework of the proposed approach. 
Since the developed transmitting boundary is formulated in the context of the finite element analysis, it is applicable to any periodic structures.

\section{References}

( 1 ) Knothe, K.L. and Grassie, S.L., Vehicle System Dynamics, Vol.22(1993), pp.209-262.

( 2 ) Abe, K. and Furuta, M., Journal of Structural Engineering, Vol.43A(1997), pp.365-372 (in Japanese).

( 3 ) Abe, K., Satou, D., Suzuki, T. and Furuta, M., Three-dimensional analysis of subway track vibrations due to running wheels, Proceedings of The International Workshop WAVE2002, (2002-9), pp.149-156.

( 4 ) Phani, A.S., Woodhouse, J. and Fleck, N.A., Journal of the Acoustical Society of America, Vol.119(2006), pp.1995-2005.

( 5 ) Abe, K., Furuya, T. and Koro, K., Journal of Applied Mechanics JSCE, Vol.10(2007), pp.1029-1036 (in Japanese).

( 6 ) Clouteau, D., Arnst, M., Al-Hussaini, T.M. and Degrande, G., Journal of Sound and Vibration, Vol.283(2005), pp.173-199.

( 7 ) Lysmer, J. and Waas, G., Journal of the Engineering Mechanics Division ASCE, EM98(1972), pp.85-105.

( 8 ) Brillouin, L., Wave propagation in periodic structures, (1953), pp.80-93, Dover Publications.

( 9 ) Lee, S. and Tanabe, T., Journal of Structural Mechanics and Earthquake Engineering JSCE, No.745(2003), pp.25-38 (in Japanese).

(10) Abe, K., Kasahara, Y. and Koro, K., Journal of Applied Mechanics JSCE, Vol.11(2008), pp.1031-1038 (in Japanese). 